Check for updates

Cite this: RSC Adv., 2017, 7, 38193

Received 16th June 2017

Accepted 21st July 2017

DOI: $10.1039 / \mathrm{c} 7 \mathrm{ra06717h}$

rsc.li/rsc-advances

\section{Mesoscopic probes in asphaltenes nanoaggregate structure: from perpendicular to paralleled orientation at the water-in-oil emulsions interface $\uparrow$}

\author{
Jinhui Chen, ${ }^{a}$ Jinliang Chen, ${ }^{\mathrm{b}}$ Chuheng Zhong, ${ }^{\mathrm{c}}$ Shouyu Chen, ${ }^{a}$ Bowen Chen, ${ }^{\mathrm{d}}$ \\ Shenwen Fang $\mathbb{D} t^{* e}$ and Wenjun Xiang ${ }^{f}$
}

It is well known that asphaltene molecules play a significant role in stabilizing emulsions of water-in-crude oil or diluted bitumen solutions. Here, the dissipative particle dynamics simulation method was employed to investigate the aggregation and orientation behaviors of asphaltene molecules at the interface of water-incrude oil emulsions at mesoscale length. Three kinds of asphaltene model molecules with different architectural structures were employed in this work. It was found that the initially disordered asphaltenes quickly self-assembled into ordered nanoaggregates consisting of several molecules, in which the aromatic rings in asphaltenes were reoriented to form nanoaggregate structures. More importantly, the nanoaggregate structure indicates that most of the stacked polycyclic aromatic planes of island architecture asphaltenes preferred to be perpendicular to the O/W interface. However, most of the stacked polycyclic aromatic planes of archipelago architecture asphaltene tend to be parallelled to the $\mathrm{O} / \mathrm{W}$ interface. Both the perpendicular and parallel nanoaggregate structures of asphaltenes form a steady protective film wrapping the water droplets which hinder the droplet-droplet coalescence. The increasing number of aliphatic chains of asphaltenes results in hindering of the $\pi-\pi$ stacking interactions of polycyclic aromatic hydrocarbons, thus asphaltenes prefer to form the parallel orientation at the O/W interface. Our results provide insights into the fundamental understanding of self-assembly mechanisms of asphaltenes at $\mathrm{O} / \mathrm{W}$ interface and the stabilization behaviors of water-in-oil emulsions with asphaltenes.

\section{Introduction}

Asphaltenes are practically defined as a solubility class soluble in some solvents, such as aromatics (benzene, toluene, etc.), but insoluble in some others, such as normal alkanes (paraffins, heptane, etc.). ${ }^{1}$ Asphaltenes are usually presented in petroleum industry and coal liquid as well mine of coalbed gas (e.g. green mine construction). The structure of asphaltenes is complex and normally assumed to be constituted by polycyclic aromatic hydrocarbons (PAHs) substituted with alkyl side chains and

${ }^{a}$ China University of Geosciences (Wuhan), Wuhan 430074, China

${ }^{b}$ Management Center of Sustainable Development Research Institute of Yellow River Delta, Dongying, Shandong, 257091, China

${ }^{c}$ Ecole Centrale de Nantes, Pays de la Loire, 44000 Nantes, France

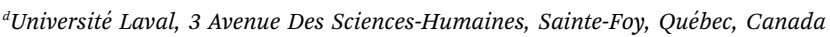

${ }^{e}$ College of Chemistry and Chemical Engineering, Southwest University of Petroleum, 8 Xindu Avenue, Xindu District, Chengdu, Sichuan 610500, P. R. China. E-mail: fangwen1228@yahoo.com.cn; Fax: +86028 83037346; Tel: +86028 83037346

${ }^{f}$ School of Chemistry and Chemical Engineering, Sichuan University of Arts and Science, Dazhou, Sichuan 635000, China

$\dagger$ Electronic supplementary information (ESI) available. See DOI: 10.1039/c7ra06717h

† Present addresses: 8 Xindu Avenue, Xindu District, Chengdu, Sichuan 610500, P. R. China. some heteroatoms, including nitrogen, sulfur, oxygen. ${ }^{2}$ Correspondingly, asphaltenes are kinds of interfacially active substances because of their polarizability and some degree of charge separation associated with heteroatoms in the alkyl chains and aromatic ring structures. ${ }^{3}$ The presence of asphaltenes is critical to many aspects of petroleum utilization, from oil recovery and transportation to refining. The formation of stable water-in-crude oil emulsions in petroleum industry can cause flow assurance problems due to high viscosity of the emulsions and cost efficient methods are required to remove the emulsified water from the emulsions. ${ }^{4}$ The water emulsified by asphaltenes, in the form of micrometer size water droplets, also enhance corrosion because of the inclusion of salt and brine in the petroleum emulsions, which reduce throughput, and also can lead to equipment failure. ${ }^{5}$ Therefore, understanding the mechanism of crude oil emulsion stabilized by asphaltenes is crucial for stabilizing desired emulsions and destabilizing undesired emulsions in petroleum industry.

Actually, typical asphaltene molecules cannot be considered to be amphiphilic with well-defined hydrophilic and hydrophobic molecular architecture. Moreover, the affinity of asphaltenes toward the $\mathrm{O} / \mathrm{W}$ interface and their ability of reducing the interfacial tension are lower than the other indigenous crude/heavy oil fractions (such as resins). ${ }^{6}$ A large 
number of studies indicate the mechanism of emulsion stabilized by asphaltenes is related to the formation of the nanoaggregate structure at $\mathrm{O} / \mathrm{W}$ interface. Several researchers ${ }^{7-9}$ have proposed that a kind of cross-linked gel phase is formed at the $\mathrm{O} / \mathrm{W}$ interface which results into hindering droplet-droplet coalescence. Building up of 3D network at the interface, the rheological properties of the asphaltenes film were modified to be non-Newtonian with yield stress (gel-like), which is responsible for the stability of crude oil emulsions. ${ }^{\mathbf{8} 9}$ The order asphaltene nanoaggregate structure, typically 7-20 nm, was observed by small-angle X-ray scattering (SAXS) and small-angle neutron scattering (SANS). ${ }^{6,10}$ Furthermore, the colloidal asphaltene aggregates as compared to individual asphaltene molecules at the $\mathrm{O} / \mathrm{W}$ interface were also thought to increase the stability of emulsions. ${ }^{\mathbf{1 1}}$ Liu Juan and co-author ${ }^{\mathbf{1 2}}$ employed molecular dynamics simulations studied the nanoaggregate structure of asphaltenes. They found most of the stacked polycyclic aromatic planes of asphaltene nanoaggregates tend to be perpendicular to the water surface. These asphaltenes adsorbed at the $\mathrm{O} / \mathrm{W}$ interface seems as a "fence" and all the fence-like nanoaggregates were twined and knitted together, which pinned them perpendicularly on the water surface to form a steady protective film wrapping the water droplets. The asphaltenes film with nanoscale aggregates at the interface is much stable and rigid. ${ }^{9,12}$ In our previous studies, it was found that the rigid mechanical film of asphaltenes originates from (i) rigid structure of polycyclic aromatic hydrocarbons (PAHs) and (ii) the $\pi-$ $\pi$ bonding interactions between the PAHs of asphaltenes. ${ }^{13}$ Moreover, a layer-by-layer assembled architecture film was formed at the W/O interface when the system in presence of polyacrylamide (PAM). This layer-by-layer assembled architecture film exhibits great viscoelasticity and rheology properties which enhances the stability of water-in-oil emulsions.

Moreover, the structure of asphaltenes also has a great influence on the nanoaggregate structure at the $\mathrm{O} / \mathrm{W}$ interface. ${ }^{\mathbf{1 4}}$ To this end many studies have been carried out on the nanoaggregate structure at the $\mathrm{O} / \mathrm{W}$ interface with different kinds of asphaltene structure. Chang Chia-Lu and co-authors ${ }^{15}$ investigated the effect of the chemical structure of amphiphiles on asphaltenes stabilization with tuning the polarity of the asphaltenes's head group and the length of the asphaltenes's alkyl tail. The effects of side-chain length on the aggregation of asphaltenes in water were studied by Jian Cuiying and coauthors. ${ }^{16}$ Hugo Santos-Silva and co-author ${ }^{17}$ investigated the effects of the $\mathrm{N}, \mathrm{O}$ and $\mathrm{S}$ heteroatoms on the nano-aggregation of asphaltene mixtures.

Ruiz-Morales Yosadara and co-author ${ }^{5}$ used the coarsegrained molecular simulations to investigate the effect of peripheral oxygen moieties in coarse-graining asphaltene on the orientation of asphaltene at the $\mathrm{O} / \mathrm{W}$ interface, they found the asphaltene orients perpendicularly at the oil water interface when the asphaltene contains oxygen atoms. Furthermore, it was observed that the nanoaggregation of asphaltene is dependent on the heteroatom on the conjugated core and is more dependent on the chain-end and conjugated core size. Hossein Rezaei and co-author ${ }^{\mathbf{1 8}}$ studied the uncharged perylene bisimide-based polyaromatic surfactant molecules, with the same polyaromatic core but with different terminal functional types at the $\mathrm{O} / \mathrm{W}$ interface. They found that the polyaromatic surfactant with the terminal functional group of aliphatic carboxylic acid absorbed more effectively on the water droplet interface in the crude oil system and can reduce the interfacial tension to facilitate the oil/water separation. Yohei Mikami investigated the nanoaggregate structure of three kinds of asphaltenes with low molecular weights ${ }^{\mathbf{1 9}}$ at the nanoscale. Though a large number of the aforementioned studies have been reported, a further understanding of the nanoaggregate structure and emulsion stability of asphaltenes at the $\mathrm{O} / \mathrm{W}$ interface is still needed, especially at mesoscopic scale. ${ }^{13}$

In this work, we employed dissipative particle dynamics (DPD) simulations for studying the nanoaggregate structures of asphaltenes at water/toluene interface with the mesoscopic scale. ${ }^{13,22}$ DPD simulations method are an excellent technology for the simulation of coarse-grained systems over considerable length and time scales up to the mesoscopic scale. ${ }^{13,20}$ DPD simulations have been performed to study static and dynamic interfacial phenomena and the self-assembly behavior of amphiphilic molecule at the interface, ${ }^{\mathbf{2 0 , 2 1}}$ including asphaltenes at liquid/ liquid interface ${ }^{22}$ and the solid/liquid interface. ${ }^{23}$ In this study, we have used three types of asphaltene molecules with different molecular weights, which was constructed from a statistical representation of the oil sample. ${ }^{24}$ The stability mechanism of water-in-oil emulsions in presence of asphaltenes was investigates by analyzing the morphologies, film thickness, order parameter and radial distribution function of the crude oil emulsion.

\section{DPD simulation}

\subsection{DPD method}

Dissipative particle dynamics (DPD) is a mesoscale particle method that bridges the gap between microscopic and macroscopic simulations. It can be used to simulate the dynamic and rheological properties of simple and complex fluids. In DPD simulation system, several molecules were usually coarsegrained as one or more coarse-grained beads, respectively. The dynamics of the elementary units which are so-called DPD beads, is governed by Newton's equation of motion $f_{i}=m \mathrm{~d} v_{i} / \mathrm{d} t$. The force between each pair of beads is a sum of a conservative force $\left(\boldsymbol{F}_{i j}^{\mathrm{C}}\right)$, a dissipative force $\left(\boldsymbol{F}_{i j}^{\mathrm{D}}\right)$, and a random force $\left(\boldsymbol{F}_{i j}^{\mathrm{R}}\right){ }^{25}$ The conservation force for non-bonded particles is defined by soft repulsion. The dissipative force corresponding to a frictional force depends on both the position and relative velocities of the beads. The random force is a random interaction between bead $i$ and its neighbor bead $j$. All forces vanish beyond a certain cutoff radius $r_{\mathrm{c}}$, whose value is usually set to one unit of length in simulations. In fact, only the conservation force is related to the intrinsic properties of the bead and it is given by the following formulas: $\boldsymbol{F}_{i j}^{\mathrm{C}}=\alpha_{i j} \omega\left(r_{i j}\right) \mathbf{e}_{i j}$, where $\alpha_{i j}=\alpha_{j i}>$ 0 , indicating that this force is always repulsive, $r_{i j}$ is the distance between beads $i$ and $j$, and $\mathbf{e}_{i j}$ is the unit vector $\left(\mathbf{r}_{i}-\mathbf{r}_{j}\right) / r_{i j}$. The function $\omega(r)$ determines the radial dependence of the force; the function is continuous, positive for $r<r_{\mathrm{c}}$ and zero for $r \geq r_{\mathrm{c}}$. An extensive description on DPD simulation can be found 
elsewhere, ${ }^{\mathbf{2 6 , 2 7}}$ more details about DPD technique also can be found in our previous studies. ${ }^{\mathbf{1 3 , 2 2 , 2 8}}$

To coarse-grain the model of asphaltenes, the bonded potentials including bond potential, angle potential, and inversion angle potential were introduced, as depicted in our previous studies. ${ }^{13,22,28}$ The bond potential experienced by bead $i$ is $f^{\mathrm{S}}=\sum_{\mathrm{b}} \frac{1}{2} C_{\mathrm{b}}\left(r_{\mathrm{b}}-r_{0}\right)^{2}$, where $r_{0}$ is the equilibrium bond length and $C_{\mathrm{b}}$ is the bond spring constant. The equilibrium bond length $r_{0}=3 \AA$ and a spring constant of $300 \mathrm{kcal}$ (mol $\left.\AA^{2}\right)^{-1}$ are assigned for all the beads of aromatic rings, while $r_{0}=$ $4.25 \AA$ and $C_{\mathrm{b}}=3 \mathrm{kcal}\left(\mathrm{mol} \AA^{2}\right)^{-1}$ are taken for all the alicyclic groups and alkyl chains. The angle potential experienced by bead $i$ due to angular interactions is $f^{\mathrm{A}}=\sum_{\mathrm{a}} k_{\mathrm{a}}\left(1-\cos \left(\theta_{\mathrm{a}}-\theta_{\mathrm{a}}^{0}\right)\right)$, where $\theta_{0}=120^{\circ}$ is the equilibrium angle, and $k_{\mathrm{a}}=300 \mathrm{kcal} \mathrm{mol}^{-1}$. Furthermore the inversion angle potentials describe the interaction arising from a particular geometry of three beads $(J, K, L)$ around a central bead I: $E=\frac{K_{0}}{2}\left(1-\cos n\left[\chi-\chi_{0}\right]\right)$, where $K_{0}=25 \mathrm{kcal} \mathrm{mol}^{-1}$ is force constant, $\chi_{0}=180^{\circ}$ is the improper torsion angle between the $K I J$ and JIL plane, and $n=2$ is the periodicity.

\subsection{Coarse-graining model}

The coarse-graining process of molecular in DPD simulation system is an essential process. As reported in our previous studies, three water molecules were taken as single bead (W bead). As presented in Fig. 1, the typical beads, B represents the moiety of aromatic rings, which is denoted by benzene molecule; $\mathrm{H}$ corresponds to the alkyl chain, which is defined as butane molecule. In DPD the mapping of groups of atoms into beads, all the beads should have same volume, the volume of a water bead is $90 \AA^{3}$ in this study. ${ }^{5}$ The volume of one butane molecule is $91.5 \AA^{3}$ and one benzene molecule is $89.4 \AA^{3}$ which are close to the volume of a water bead. ${ }^{29}$ The oil phase is toluene which is clustered into two different coarse-grained particles with $\mathrm{B}$ bead and $\mathrm{H}$ bead. The fused aromatic rings is constructed by creating the rigid sheet of the hexa-particle ring. ${ }^{13,28}$ As shown in Fig. 1, $\mathrm{T}$ bead is the functional group containing heteroatoms, we take thiourea as $\mathrm{T}$ bead which represent the polar groups in asphaltene structure..$^{\mathbf{1 3 2 8}}$

All these coarse-graining models in simulation systems were used and verified. ${ }^{\mathbf{1 3 , 2 8}}$ Three different types of asphaltene models were performed to study the nanoaggregate structure of asphaltenes and their stabilization behaviors of water-in-oil emulsions, as presented in Fig. 1e. These kinds of asphaltene models were reported to study the aggregation of asphaltenes. ${ }^{24,28}$ Because of the complexity and diversity of asphaltenes in petroleum industry, different model compounds have been employed in various studies. However, the island architecture and archipelago architecture are the widely accepted model. The island architecture, as shown with Model A in Fig. 1e, is usually shaped "like your hand" with a single polycyclic aromatic hydrocarbon core (like palm), which has some aliphatic chains (like fingers). Another widely accepted model for asphaltenes is an archipelago architecture, which was thought to be connected with several polycyclic aromatic hydrocarbon core, as shown with Model B and Model $C$ in Fig. 1e. Nowadays, it remains dubious regarding the archipelago models. Mullins ${ }^{2,24}$ insisted that their large relative molar mass and colorless character disagree with observations from their experiments. The archipelago model molecules of asphaltene were constructed deliberately for comparison with the island models in this paper. Generally, the archipelago architecture model is more applicable to heavy or ultra-heavy oil rather than to light crude oil. The molecular weight of asphaltenes: Model A (about $1052.85 \mathrm{~g} \mathrm{~mol}^{-1}$ ) $>$ Model B (about $2021.93 \mathrm{~g} \mathrm{~mol}^{-1}$ ) $>$ Model $C$ (about $3248.55 \mathrm{~g} \mathrm{~mol}^{-1}$ ).

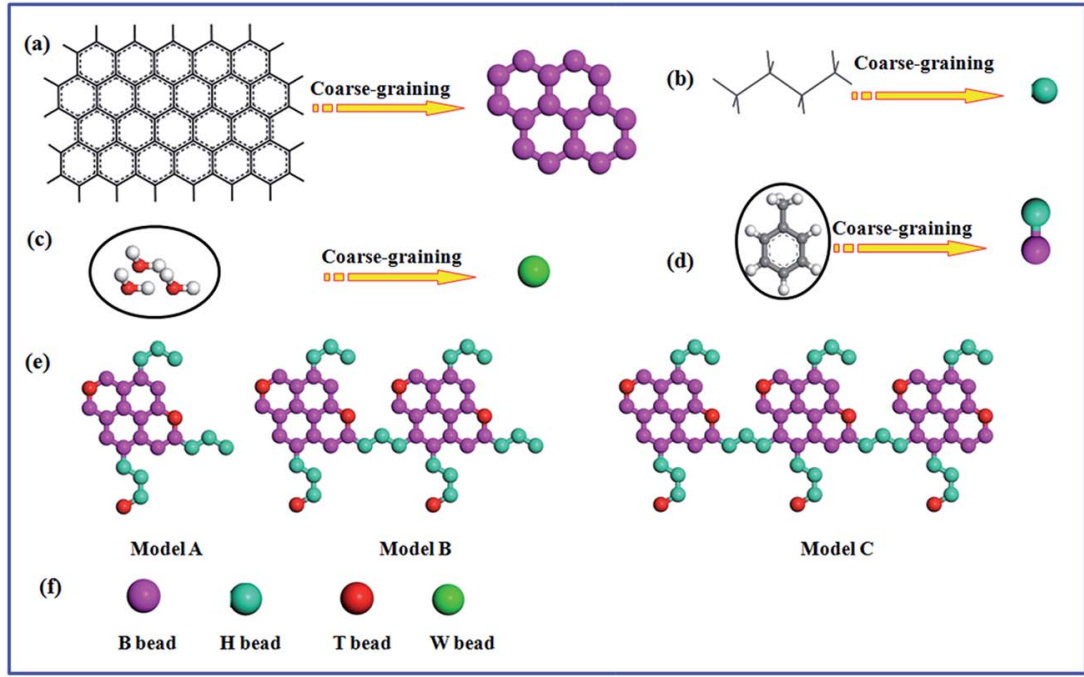

Fig. 1 Coarse-grained model molecules of (a) fused aromatic rings (or polycyclic aromatic hydrocarbons), (b) alkyl chain, (c) water molecules, (d) toluene, (e) asphaltenes model architecture: Model A, Model B and Model C, (f) corresponds to the schematic representation of coarse-grained beads in simulations. Model $A$ is island architecture and Model $B$ and Model $C$ are archipelago architecture. 


\subsection{DPD parameters and simulation details}

The bead-bead interaction parameters used in this paper are calculated from the Hansen solubility parameters. ${ }^{5,28}$ Hansen solubility parameters were developed by Charles M. Hansen to predict if one material will dissolve in another and form a solution which has been used to understand issues of solubility dispersion diffusion. ${ }^{29}$ Moreover, Hansen solubility parameters can be developed to calculate the bead-bead interaction parameters in immiscible liquid/liquid system which has been validated to produce ideal interfacial tensions in experiment method.$^{30}$ Hansen solubility parameters were also used to calculate the bead-bead interaction parameters in crude oil system. More detailed bead-bead interaction parameters in this study can be found in ESI. $\dagger$ The diffusion coefficient and interlayer distance of asphaltenes in toluene solution were calculated to validate the DPD calculations, as shown in ESI. $\dagger$ From the Fig. S1, $\uparrow$ the diffusion coefficient of diluted asphaltene in toluene is $3.41-5.73 \times 10^{-10} \mathrm{~m}^{2} \mathrm{~s}^{-1}$ which is consistent with the experimental results $2.2-6.3 \times 10^{-10} \mathrm{~m}^{2} \mathrm{~s}^{-1} \cdot{ }^{31}$ Moreover, the interlayer distance value from DPD simulations is about $5.05 \AA$ in our calculation, which is slightly larger than the experimental results (about $3.55 \AA$ ). ${ }^{32}$ On the basis of these results, it is believed that the bead-bead interaction parameters used in the simulations could well reflect the authentic physical properties of petroleum systems.

In this study, DPD simulations were performed using the Mesocite module embedded in the Materials Studio 6.1 package from Accelrys, Inc. ${ }^{33}$ All the simulations were performed in a cubic box with a size of $100 \times 100 \times 100 R_{\mathrm{c}}{ }^{3}$ with periodic boundary conditions at three direction. The simulated temperature is set as $298 \mathrm{~K}$. To simulate the water-in-crude oil emulsions, the cubic box was divided into oil phase and water phase, respectively. And water phase is a sphere with radius of $30 R_{\mathrm{c}}$ in the center of box, as reported in ref. 13 . The left part of the box is oil phase. The water molecules and toluene molecules as well as asphaltene molecules were randomly filled into oil phase and water phase, respectively. The concentration of asphaltenes is defined as the number ratio of asphaltenes beads to total beads in the oil phase. It has been shown experimentally that emulsions stabilized by individual asphaltene molecules are of little significance, when compared to stabilization by colloidally dispersed asphaltenes. ${ }^{19}$ In this study, different numbers of asphaltene molecules ranging from 56 to 94 were used to simulate the water-in-crude oil emulsions which is much higher than the number of asphaltenes used in MD simulations. The total number of beads is $1.2428 \times 10^{4}$ in the box when the density of all systems is set to 3.0 in reduced units. A total of 30.16 ns DPD simulation time were carried out with a time step $\Delta t=0.005 \tau$, where $\tau$ is time scale, $3.0158 \mathrm{ps}^{34}$

\section{Results and discussion}

\subsection{W/O emulsions morphology}

3.1.1 Dynamic behavior of nanoaggregation at the interface. The initial configurations of asphaltenes at the water-in-oil emulsions were randomized with the tool of Amorphous Cell in
Materials studio. ${ }^{33}$ In the island architecture asphaltenes (Model A) case, the molecular behavior at the toluene/water interface at various time intervals was presented in Fig. 2. From the snapshots (Fig. 2), it was found that the initial disordered asphaltene molecules quickly self-assembled into ordered structure at the $\mathrm{O} / \mathrm{W}$ interface when the system reached its equilibrium indicated. From the Fig. 2, it seems that the self-aggregation of asphaltenes (the number ranges from 2-6) is a first step in the formation of precipitated asphaltene particles at the $\mathrm{O} / \mathrm{W}$ interface. Rod-like hierarchical structural nanoaggregate consisting of a few asphaltene molecules (ranges from 12 to 18) was formed, as shown in Fig. 2f. The nanoaggregates of asphaltenes at the $\mathrm{O} / \mathrm{W}$ interface generally consist a few molecules with the molecular PAHs face-to-face or $\pi-\pi$ stacking each other. More importantly, it is interesting to find that most of the stacked aromatic ring sheets preferred to be perpendicular to the water surface. Moreover, the stacked structure remained essentially stable after $4.52 \mathrm{~ns}$.

The aggregation and orientation of the archipelago architecture asphaltenes at the $\mathrm{O} / \mathrm{W}$ interface were also investigated. As shown in Fig. 3, some similar results of adsorption process for the asphaltenes of Model B were observed. However, most of the stacked aromatic ring sheets preferred to be paralleled to the water surface. Only a small part of asphaltenes of Model B tend to be perpendicular or slope to the $\mathrm{O} / \mathrm{W}$ interface. Actually, this orientation of asphaltenes at the $\mathrm{O} / \mathrm{W}$ interface was also observed in the asphaltenes of Model $C$, as shown in Fig. S2. $\dagger$ Compared with the asphaltenes of Model A, the transition of orientation at the $\mathrm{O} / \mathrm{W}$ interface happened in asphaltenes of Model $B$ and Model $C$ was induced by architecture structure of asphaltenes. In the island architecture asphaltenes case (Model $A$ ), the asphaltenes reoriented themselves to be perpendicular with the water surface were reported in the previous molecular dynamics simulations. ${ }^{19}$ The interfacial behavior of island architecture asphaltenes at the $\mathrm{O} / \mathrm{W}$ interface in our study is in good agreement with the previous simulation results at nanoscale. ${ }^{35,36}$ However, the paralleled orientation of archipelago architecture asphaltenes at the interface was firstly observed at mesoscopic scale owing to the advantages provided by DPD simulation, such as the simulation of coarse-grained systems over long length and time scales than MD simulation. Different kinds of island architecture of asphaltenes (molecular weights are lower than $800 \mathrm{~g} \mathrm{~mol}^{-1}$ ) were investigated using MD simulations with the limited simulation time (lower than $6 \mathrm{~ns}$ ). It is also observed that the water droplet in oil is wrapped tightly by asphaltenes when the system reaches equilibrium, which produces asphaltene protective films hindering the dropletdroplet coalescence.

3.1.2 Effects of concentrations. We also study the effects of concentrations of asphaltenes on the morphology of emulsions. Form the snapshots (Fig. 4), it was found that asphaltenes of Model A preferred to be perpendicular to the water surface with the concentrations range from the $15 \%$ to $25 \%$. Except for the asphaltenes of Model B at higher concentrations (e.g. 25\%), the asphaltenes of Model $B$ and Model $C$ preferred to be paralleled to the water surface. We also calculated the temporal evolution of angles between the asphaltene aromatic plane and the $\mathrm{O} / \mathrm{W}$ 

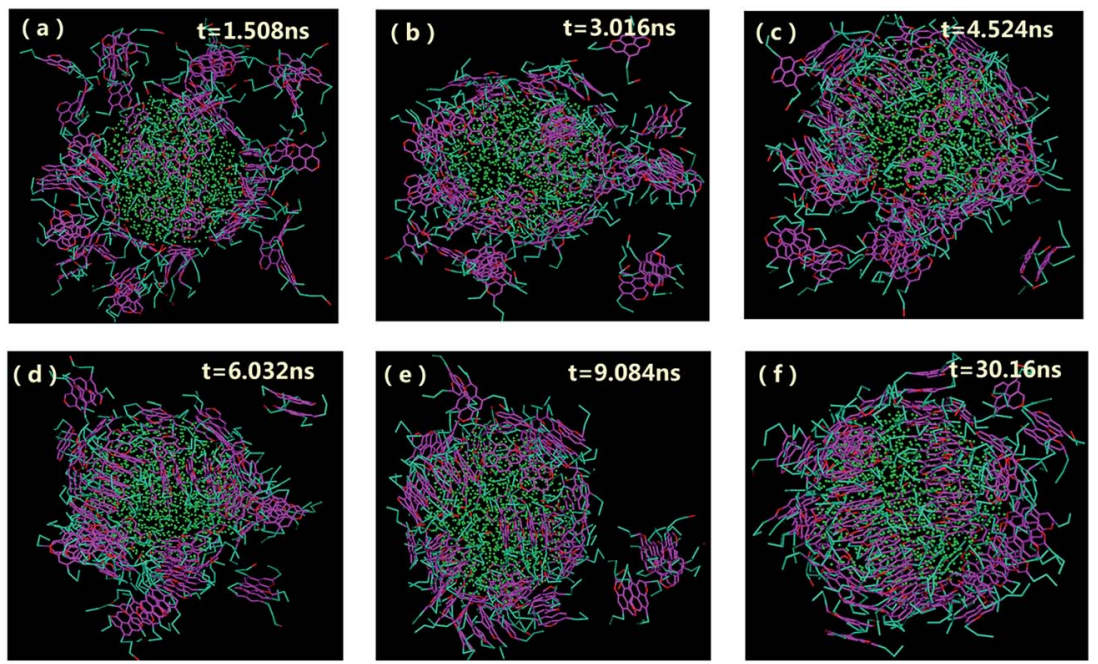

Fig. 2 Morphologies of water-in-oil emulsions at different simulation time with the concentrations of $20 \%$ asphaltenes of Model A. Different beads in simulations are represented by different colors as can be illustrated from Fig. 1f (the same as below). The toluene molecules are suppressed for clarity.
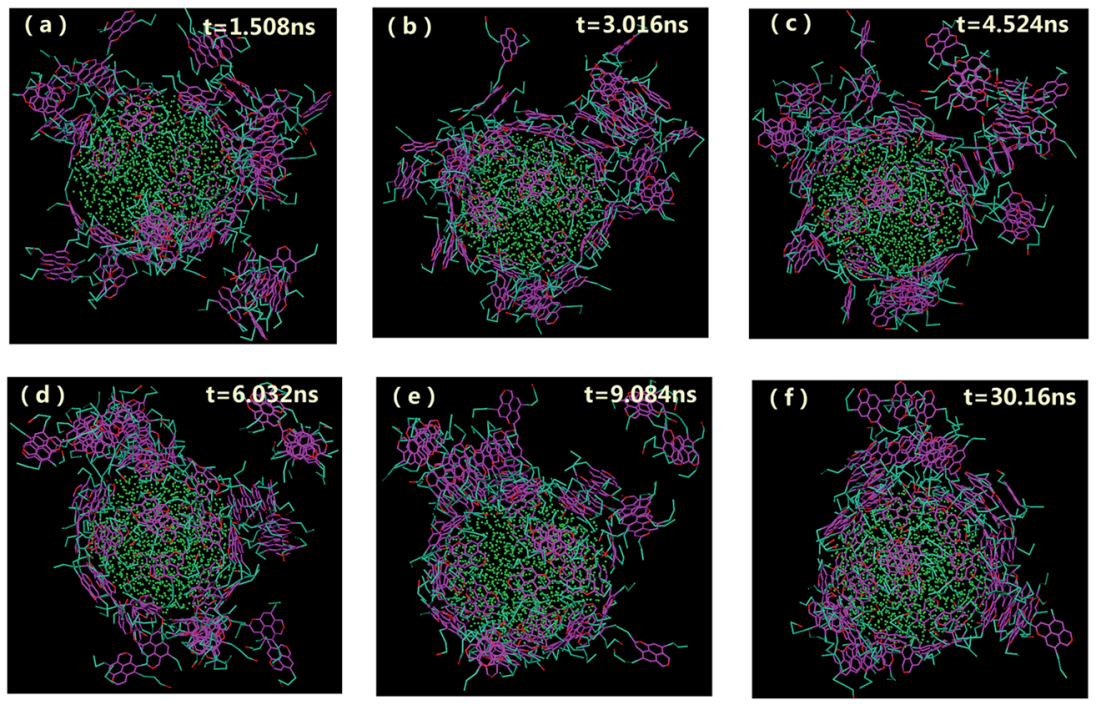

Fig. 3 Morphologies of water-in-oil emulsions at different simulation time with the concentrations of $20 \%$ asphaltenes of Model $B$. Different beads in simulations are represented by different colors as can be illustrated from Fig. 1f (the same as below). The toluene molecules are suppressed for clarity.

interface for different systems, as shown in the Fig. 5. In the asphaltenes of Model $A$ case (Fig. 5a), this angle mainly range at $85 \pm 5^{\circ}$ with the highest value is 0.55 . This means that the perpendicular structure is stable in the asphaltenes of Model $A$ case. Except for the asphaltenes of Model $B$ at concentrations of $25 \%$, the angles of asphaltenes of Model $A$ and $C$ were distribution in $5 \pm 5^{\circ}$ which shows that the asphaltenes of Model $A$ and $C$ can adopt a paralleled structure with respect to the oilwater interface. All these results can be reflected from the Fig. 4 .

\subsection{Film properties}

3.2.1 Film thickness. Recent investigations have focused on measurable mechanical properties of the films by interfacial tensiometry and rheological techniques. ${ }^{37,38}$ The film thickness is an important interfacial physical property which can provides a quantitative measure for the size of the interface. The relative density profiles are given in Fig. S3 of ESI. $\uparrow$ The density profile is obtained by calculating the number of particles with dividing the volume into 200 bins along as $X$-direction. According to the density profiles, the interfacial thickness is calculated by the "90-10" criterion, which is defined as the distance along the interface over which the densities of oil from $90 \%$ to $10 \%$ of their bulk value. ${ }^{39}$ As shown in Fig. S3, $\uparrow$ relative density profiles for asphaltenes distribute in common boundary of density profiles for aqueous phase and oil phase which indicates the asphaltenes adsorb at the $\mathrm{W} / \mathrm{O}$ interface due to the amphiphilic 

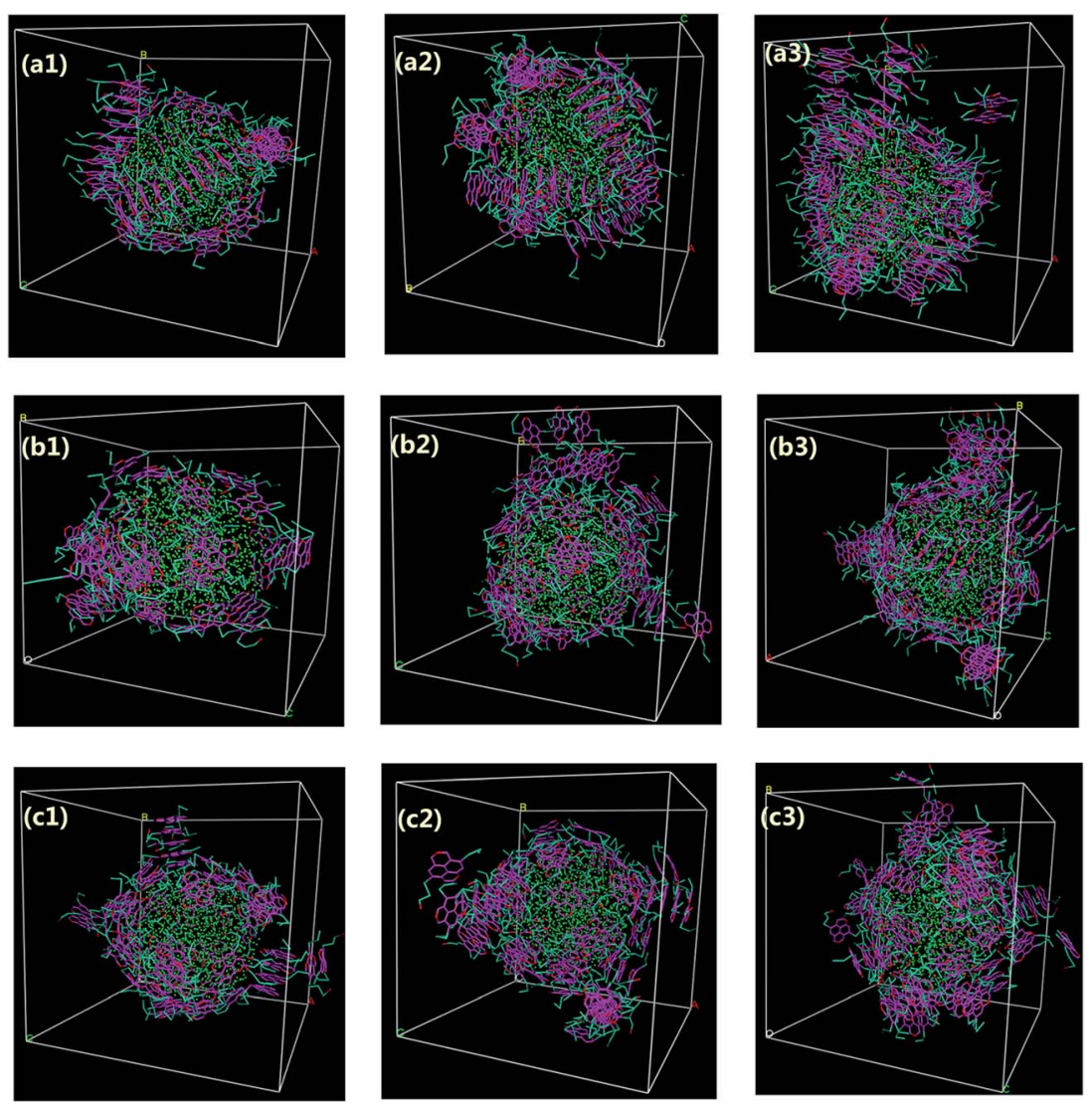

Fig. 4 Morphologies of water-in-oil emulsions at different concentrations with different asphaltene models. Asphaltenes of Model $A$ were used: (a1) $15 \%$, (a2) $20 \%$, (a3) 25\%; asphaltenes of Model B were used: (b1) 15\%, (b2) 20\%, (b3) 25\%; asphaltenes of Model C were used: (c1) $15 \%$, (c2) $20 \%$, (c3) $25 \%$. The toluene molecules are suppressed for clarity.

nature of asphaltenes. The calculated film thickness is presented in Fig. 6. Compared with orientation of paralleled structure at the interface, the film thickness formed by asphaltenes with perpendicular orientation at the interface is much bigger. Based on the change of film thickness, this nanoaggregate structure transformation of asphaltenes was verified: from the nanoaggregate to thin-film formation at the interface. Moreover, the increasing asphaltenes concentration leads to a pronounced expansion of the interfacial thickness. The calculated film thickness are range from 32.1 to $45.9 \mathrm{~nm}$ which slightly less than experimental dada $40-90 \mathrm{~nm} .^{40}$ The asphaltenes with higher concentrations in experiments may lead to a higher film thickness.

3.2.2 Nanoaggregate structure at the interface. To further characterize the nanoaggregate structures of asphaltenes at the $\mathrm{O} / \mathrm{W}$ interface, the radial distribution function $g(r)$ was introduced in this paper. The radial distribution function $g(r)$ was computed for all pairs of beads or centroids in the set which were closer than the cutoff value. The radial distribution function could be calculated using following equation ${ }^{41}$

$$
g_{i j}(r)=\frac{\left\{\Delta N_{i j}(r \rightarrow r+\Delta r)\right\} V}{4 \pi r^{2} \Delta r N_{i} N_{j}}
$$

where $\left\{\Delta N_{i j}(r \rightarrow r+\Delta r)\right\}$ was the ensemble averaged number of $j$ around $i$ within a shell from $r$ to $r+\Delta r, V$ was the system volume, $N_{i}$ and $N_{j}$ were number of $i$ and $j$, respectively.

The radial distribution function in this study was calculated for a cut-off radius of $50 \AA$ and an interval distance of $0.05 \AA$, is shown in Fig. 7. In the asphaltenes of Model A case, it is found that the multi-peaks locates at $5.4 \AA, 10.2 \AA, 15.1 \AA, 21.5 \AA, 27.4$ $\AA$, respectively. These results depicted that the asphaltene molecules aggregated spontaneously at the interface and formed nanoaggregates with the aromatic rings sheet face-toface or $\pi-\pi$ stacking together. These small peaks reflect that the asphaltenes films adsorbed at the $\mathrm{O} / \mathrm{W}$ interface are multilayers due to the intensively $\pi-\pi$ bonding between PAHs of asphaltenes which indicates the asphaltene films are much more stable. The nanoaggregates of asphaltenes at the $\mathrm{O} / \mathrm{W}$ interface were also observed from experiment results ${ }^{42,43}$ and MD simulations. ${ }^{44}$ As reported in the previous studies, the nanoaggregates range from 7 to $20 \mathrm{~nm}$ in characteristic dimension while the aggregate number ranges from 6 to $14 . .^{45}$ In our simulation results, the aggregates size of 6-22 $\mathrm{nm}$ and aggregate number of 7-13 were observed which are qualitatively in good agreement with the previous simulation results. The multi-peaks phenomenon was also observed in the asphaltenes of Model $B$ and $C$, as shown in Fig. 7b and c. However, the phenomenon of periodic peaks in the radial distribution 

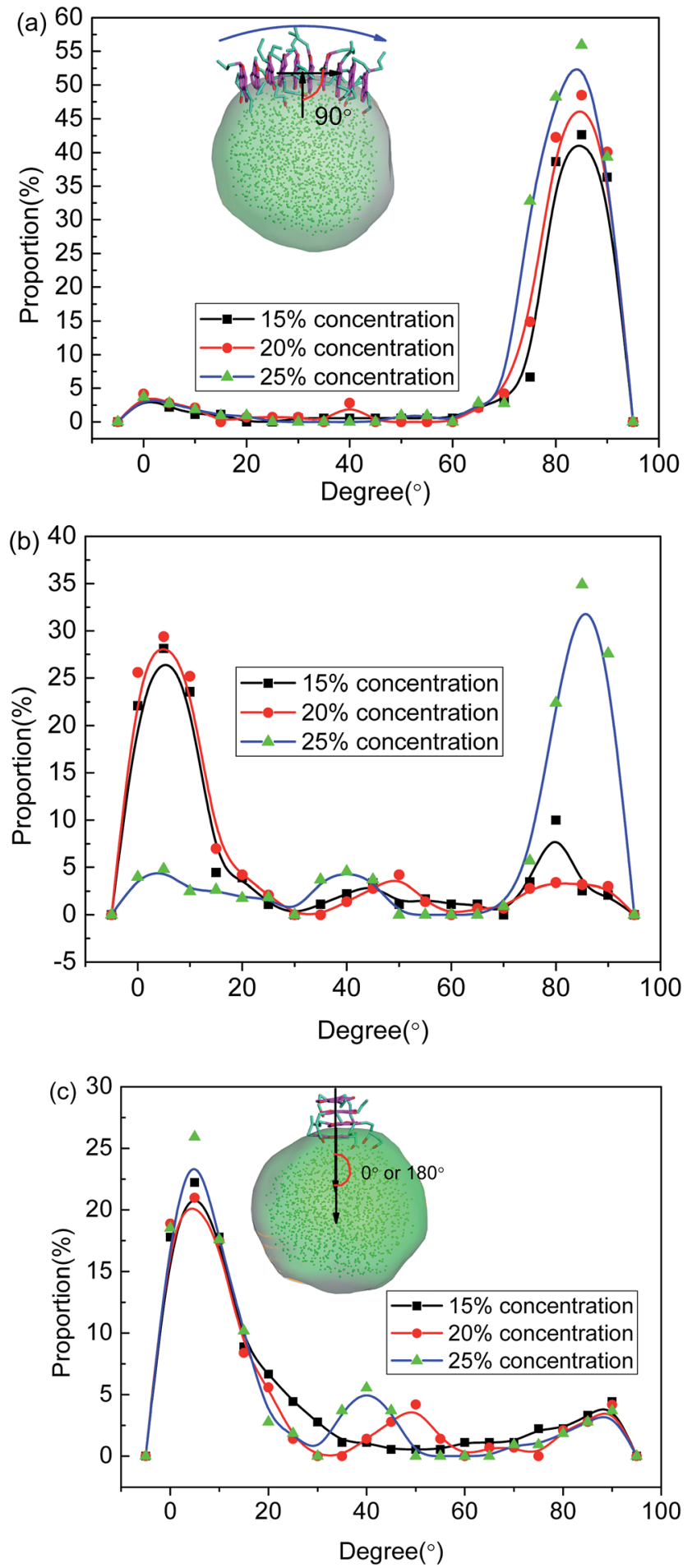

Fig. 5 Temporal evolution of angles between the asphaltene aromatic plane and the O/W interface for the systems including (a) asphaltene molecule of Model A, (b) asphaltene molecules of Model B and (c) asphaltene molecules of Model $C$. The angle is defined on the basis of the normal of the interface and the normal of the aromatic plane of the island-type asphaltene molecule.

function for the asphaltenes of Model $B$ and $C$ is not obvious than the asphaltenes of Model A. Moreover, the values of peaks for the asphaltenes of Model $B$ and $C$ are smaller than the asphaltenes of Model A. These results presented the $\pi-\pi$

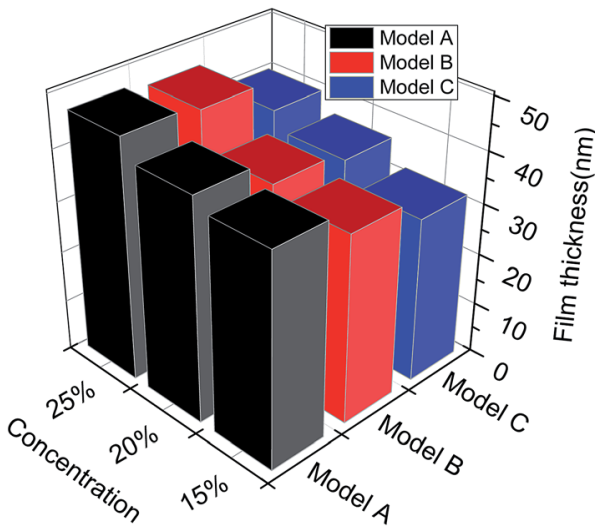

Fig. 6 The film thickness for different asphaltene models at different concentrations.

interactions between PAHs of asphaltenes gradually become weaken. Thus, the smaller aggregates size 5-12 $\mathrm{nm}$ and aggregate number 3-7 were also observed, as shown in the Fig. 3.

To further investigate the preferred distribution and orientation of asphaltenes at $\mathrm{O} / \mathrm{W}$ interface, a quantitative analysis on the orientation of the asphaltenes was conducted by calculating the radial distribution function $g(r)$ for different beads in asphaltene structure. As depicted in Fig. 8. The peaks of radial distribution function between $\mathrm{W}$ beads and $\mathrm{T}$ beads are much higher than the radial distribution function between $\mathrm{W}$ beads and $\mathrm{B}$ beads as well as $\mathrm{H}$ beads. These results are due to the fact that the $\mathrm{W}$ beads containing some heteroatoms have stronger affinity for water molecules. What's more, some smaller peaks of radial distribution function between $\mathrm{W}$ beads and $\mathrm{T}$ beads (marked by green color) were found in Fig. 8. Tough these smaller peaks are not obvious, these smaller peaks can be only found in the radial distribution function between $\mathrm{W}$ beads and $\mathrm{T}$ beads which illustrates the asphaltene molecules orients at $3.2 \AA$ from the water surface. In the asphaltenes of Model A case, there are two peaks in the radial distribution function between $\mathrm{W}$ beads and $\mathrm{T}$ beads which is resulted from the perpendicular orientation of asphaltenes at the interface (see the illustration of Fig. 8a). The phenomenon of multi-peaks was observed in the asphaltenes of Model B and C. However, these results are due to the paralleled orientation of asphaltenes at the interface, as shown in illustration of Fig. $8 \mathrm{~b}$ and c. More importantly, the aliphatic chains without heteroatoms tend to stretch into toluene region with the aliphatic chains with heteroatoms are perpendicular to water region. Jian Cuiying et al. ${ }^{16}$ proposed that the aliphatic chains hinder the formation of parallel $\pi-\pi$ stacking structures of the PHAs while also favoring aggregation through hydrophobic association with enough long side chains. As presented in Fig. 8, the values of peaks between $\mathrm{W}$ beads and $\mathrm{H}$ beads are smallest in all the beads of asphaltenes, which indicates the side chains of asphaltenes hinder the formation of parallel stacking structures of the polyaromatic cores. In this study, the aliphatic chains of asphaltenes equivalent to dodecane molecule which hinder the $\pi-\pi$ stacking interactions of PHAs. Moreover, the number of aliphatic chains in archipelago architecture asphaltenes is 


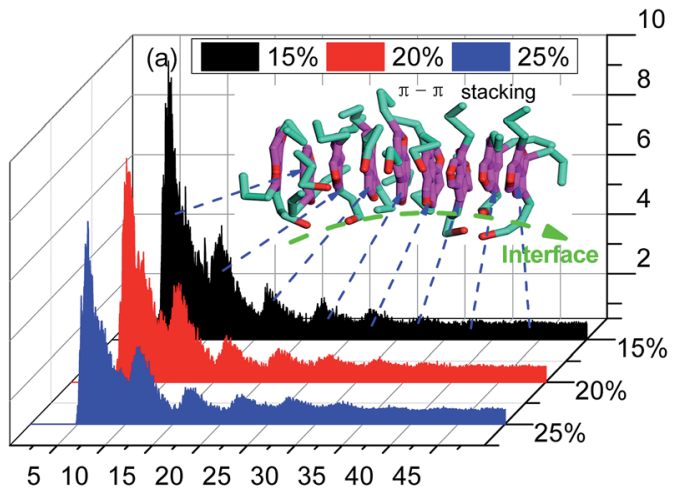

$r(\AA)$
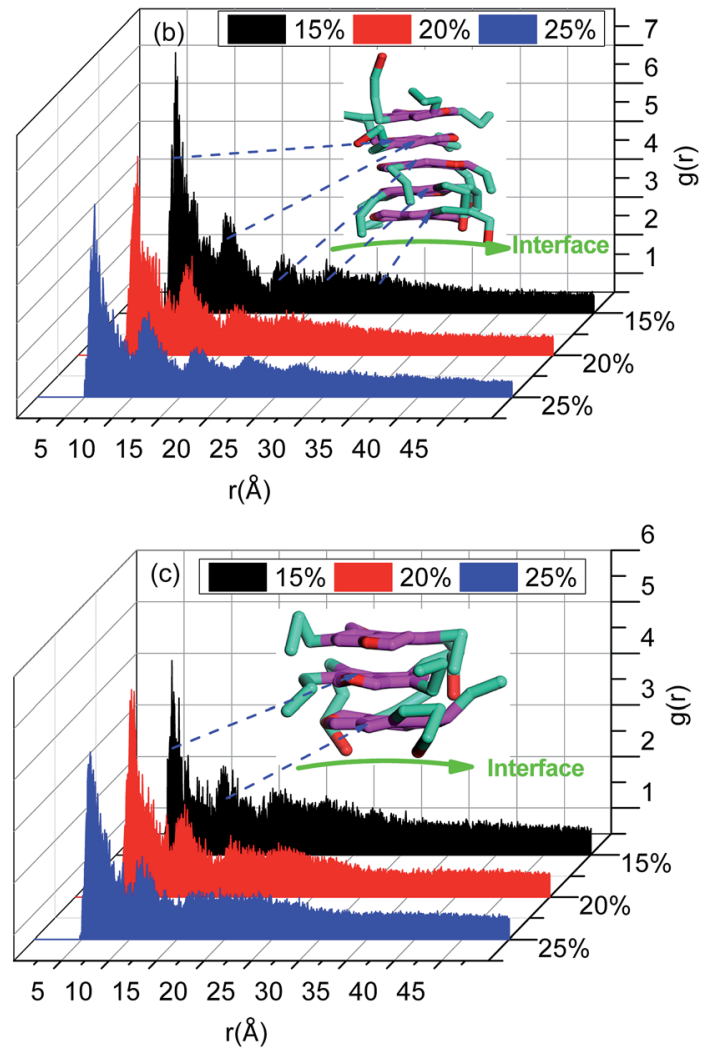

Fig. 7 The radial distribution function $g(r)$ for asphaltenes in different simulation systems including (a) asphaltene molecule of Model $A$, (b) asphaltene molecules of Model $B$ and (c) asphaltene molecules of Model C.

bigger than the island architecture asphaltenes. Actually, this result also has an impact on the nanoaggregate structure of asphaltenes at the $\mathrm{O} / \mathrm{W}$ interface. Because of the increasing aliphatic chains, the PHAs of archipelago architecture asphaltenes preferred parallelling to the $\mathrm{O} / \mathrm{W}$ interface which increasing the areas of contact with water droplet while decreasing the probability of $\pi-\pi$ stacks of PHAs in the oil phases. Consequently, the architecture structure of asphaltenes influenced the nanoaggregate structure configuration of at the $\mathrm{O} / \mathrm{W}$ interface.

To have a better understanding on the aggregation of asphaltenes at the $\mathrm{O} / \mathrm{W}$ interface, the nanoaggregate structures
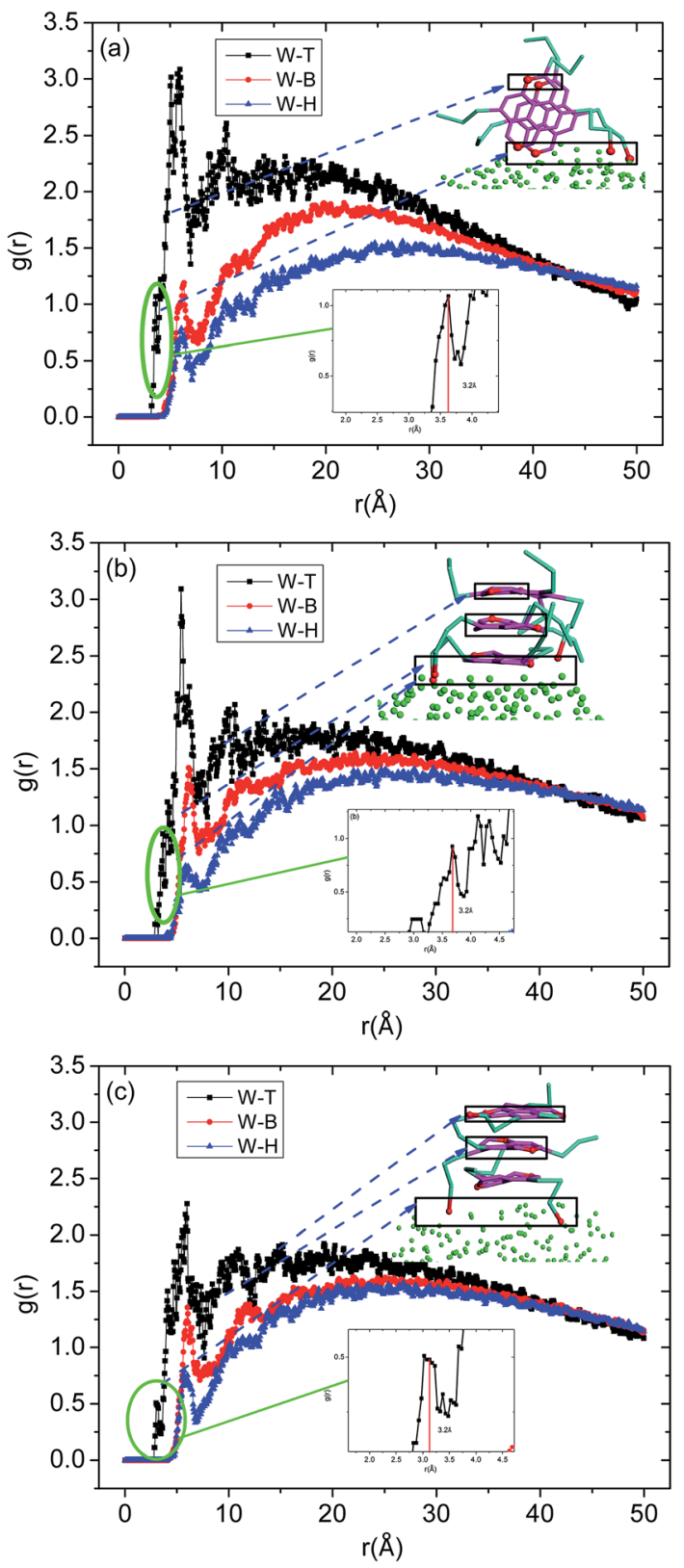

Fig. 8 The radial distribution function $g(r)$ for different beads in asphaltenes structure including (a) asphaltene molecules of Model A, (b) asphaltene molecules of Model $B$ and (c) asphaltene molecules of Model $C$. The concentrations of asphaltenes are $20 \%$. The definition of beads can be found in Fig. 1.

were analyzed using order parameter $(P)$. In dynamic simulation processes, an order parameter could be monitored to indicate the changes occurring in the molecular structures, and could thus yielded characteristics of the phase separation and compressibility. The order parameter $(P)$, which was the mean squared deviation from homogeneity for a particular species (A) in volume $V$, was defined as: ${ }^{46,47}$

$$
P_{\mathrm{A}}=\left\langle\left(\eta_{\mathrm{A}}-\eta_{\mathrm{A}}^{0}\right)^{2}\right\rangle
$$



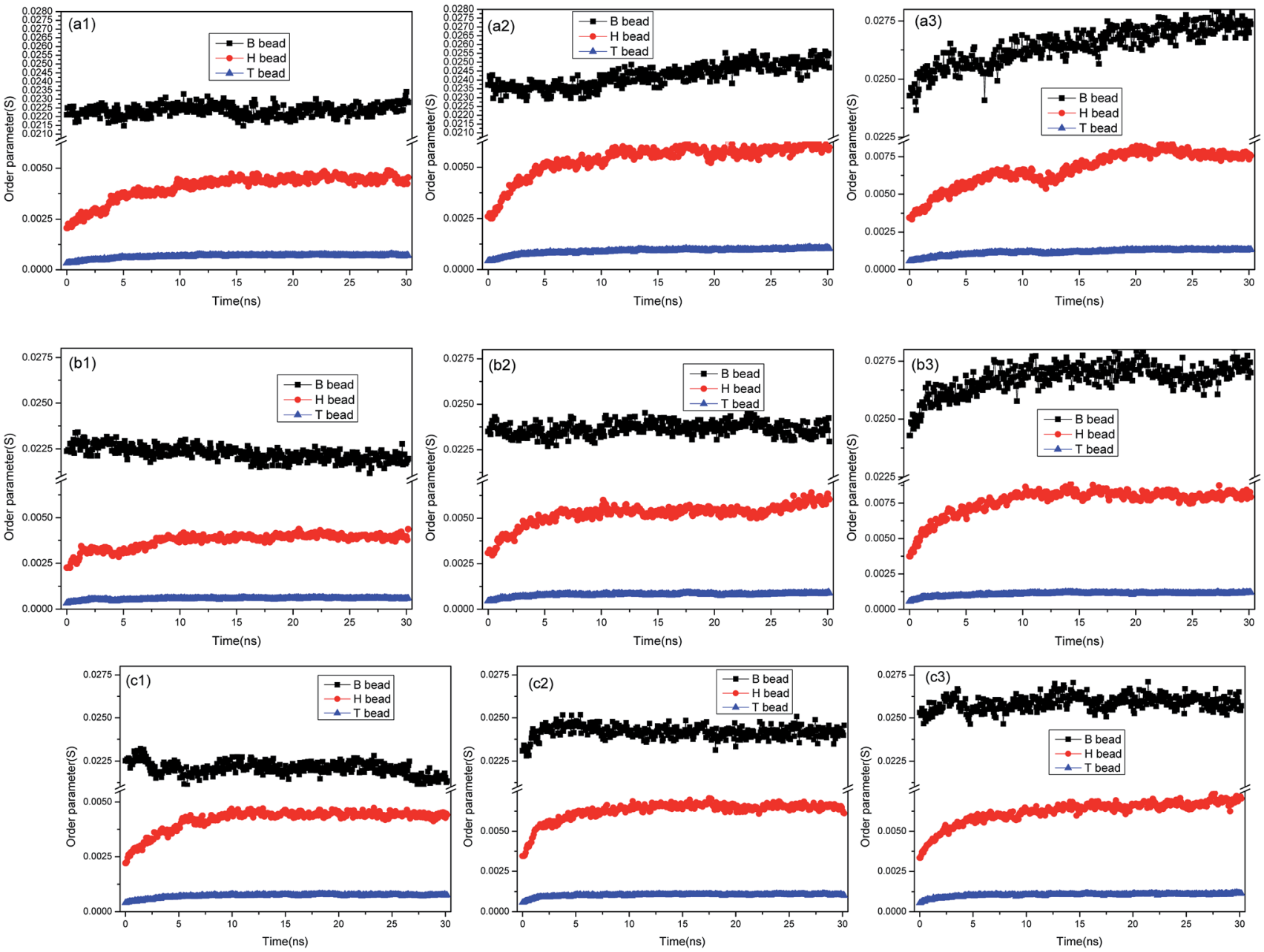

Fig. 9 Order parameter versus simulation the time for different asphaltene models at different concentrations. The asphaltenes of Model $A$ were used: (a1) 15\%, (a2) 20\%, (a3) 25\%; the asphaltenes of Model B were used: (b1) 15\%, (b2) 20\%, (b3) 25\%; the asphaltenes of Model C were used: (c1) $15 \%$, (c2) $20 \%$, (c3) $25 \%$.

where $\eta_{\mathrm{A}}^{0}$ was the overall volume fraction of species $\mathrm{A}$, and $\eta_{\mathrm{A}}$ was the local volume fraction of species A; note that both quantities were dimensionless in DPD simulations. Therefore, small values for $P_{\mathrm{A}}$ indicated a homogeneous system, and large values suggested strong phase separation.

The order parameter versus simulation the time for different asphaltene models at different concentrations were presented in Fig. 9. As time progress, the order parameters of asphaltenes at the $\mathrm{O} / \mathrm{W}$ interface increase, and finally reach platform. These results showed the asphaltenes films adsorbed at the $\mathrm{O} / \mathrm{W}$ interface become more ordered and stable which can be reflected from the Fig. 2. The concentrations of asphaltenes in simulation systems also have an influence on the values of order parameters. From the Fig. 9, we can found the order parameters of asphaltenes continuously increase with the increase of concentrations. Thus, higher concentration of asphaltenes could result in the formation of more ordered and stable interfacial films which acts a barrier to droplet coalescence. Moreover, it is also observed that the order parameter for B beads is bigger than $\mathrm{H}$ and $\mathrm{T}$ beads owing to the multi-layered structure of PAHs. These results also depicted that it is the multi-layered structure of PAHs or $\pi-\pi$ staking of PAHs attributes to the stable asphaltene films rather than the alkyl chains with heteroatoms.

\section{Conclusions}

The nanoaggregate structure of asphaltenes at the $\mathrm{O} / \mathrm{W}$ interface has been studied for decades by different experimental technologies, however, very few studies have been performed on investigating the aggregation and orientation behaviors of asphaltene molecules with different architecture structure at the $\mathrm{O} / \mathrm{W}$ interface with mesoscale length. Simulation results confirm that the asphaltenes nanoaggregate structures at waterin-oil emulsions interface were majorly mediated by architecture structure as changed from perpendicular to paralleled orientation at the interface with changing from the island architecture to archipelago architecture of asphaltenes. From the simulation results, it is found that the interfacial activity or the driven force of adsorption at interface is attributed from the heteroatoms in asphaltene structures while the formation of nanoaggregate structures at the interface is resulted from the 
$\pi-\pi$ bonding interactions. This nanoaggregate structure transformation of asphaltenes also results into thin-film formation with paralleled orientation of asphaltenes at the interface. From our study, it seems that the integrity of the thin film is formed with an increasing amount of asphaltenes, which might indicate an enhanced stability of water-in-oil emulsions. The resultant effects on the emulsion, including elastic and viscous contributions, will be discussed in the near future.

\section{Acknowledgements}

This study was supported by Faculty of Earth Resources, China University of Geosciences (Wuhan) and Ecole Central Nantes and Université Laval and the project of integrated exploration project from Chinese Development Research Center of Geological Survey, key fundamental geological study on the integrated exploration area of Baiyinchang polymetallic district, Gansu province (12120114050101). This study was also supported by Special fund for public welfare of the Ministry of Land and Resources: application demonstration and scientific research base construction of three dimensional integrated exploration technology of metal mineral resources (2010110026). Besides, we acknowledge the supports from the Henan coalbed methane development and utilization company Ltd. Zhengzhou and Mine companies in Jiaozuo and Yongcheng and Hebi.

\section{References}

1 O. C. Mullins, H. Sabbah, J. Eyssautier, A. E. Pomerantz, L. Barré, A. B. Andrews, Y. Ruiz-Morales, F. Mostowfi, R. McFarlane, L. Goual, R. Lepkowicz, T. Cooper, J. Orbulescu, R. M. Leblanc, J. Edwards and R. N. Zare, Advances in Asphaltene Science and the Yen-Mullins Model, Energy Fuels, 2012, 26, 3986-4003.

2 H. Groenzin and O. C. Mullins, Molecular size and structure of asphaltenes from various sources, Energy Fuels, 2000, 14(3), 677-684.

3 Z. Hosseini-Dastgerdi, S. A. R. Tabatabaei-Nejad, E. Khodapanah and E. Sahraei, A comprehensive study on mechanism of formation and techniques to diagnose asphaltene structure; molecular and aggregates: a review, Asia-Pac. J. Chem. Eng., 2015, 10(1), 1-14.

4 J. G. Speight, Petroleum Asphaltenes-Part 1: Asphaltenes, Resins and the Structure of Petroleum, Oil Gas Sci. Technol., 2004, 59(5), 467-477.

5 Y. Ruiz-Morales and O. C. Mullins, Coarse-grained molecular simulations to investigate asphaltenes at the oil-water interface, Energy Fuels, 2015, 29(3), 1597-1609.

6 P. Tchoukov, F. Yang, Z. Xu, T. Dabros, J. Czarnecki and J. Sjöblom, Role of asphaltenes in stabilizing thin liquid emulsion films, Langmuir, 2014, 30(11), 3024-3033.

7 G. Andreatta, C. C. Goncalves, G. Buffin, N. Bostrom, C. M. Quintella, F. Arteaga-Larios, E. Pérez and O. C. Mullins, Nanoaggregates and structure-function relations in asphaltenes, Energy Fuels, 2005, 19(4), 12821289.
8 H. W. Yarranton, H. Hussein and J. H. Masliyah, Water-inHydrocarbon Emulsions Stabilized by Asphaltenes at Low Concentrations, J. Colloid Interface Sci., 2000, 228(1), 52-63; P. K. Kilpatrick, Water-in-crude oil emulsion stabilization: Review and unanswered questions, Energy Fuels, 2012, 26(7), 4017-4026.

9 P. Tchoukov, F. Yang, Z. Xu, T. Dabros, J. Czarnecki and J. Sjöblom, Role of asphaltenes in stabilizing thin liquid emulsion films, Langmuir, 2014, 30(11), 3024-3033.

10 L. Barre, J. Jestin, A. Morisset, T. Palermo and S. Simon, Relation between nanoscale structure of asphaltene aggregates and their macroscopic solution properties, Oil Gas Sci. Technol., 2009, 64, 617-628.

11 D. Fenistein, L. Barre, D. Broseta, D. Espinat, A. Livet, J.-N. Roux and M. Scarsella, Viscometric and neutron scattering study of asphaltene aggregates in mixed toluene/ heptane solvents, Langmuir, 1998, 14, 1013-1020.

$12 \mathrm{~J}$. Liu, Y. Zhao and S. Ren, Molecular dynamics simulation of self-aggregation of asphaltenes at an oil/water interface: formation and destruction of the asphaltene protective film, Energy Fuels, 2015, 29(2), 1233-1242.

13 M. Duan, X. Song, S. Zhao, S. Fang, F. Wang, C. Zhong and Z. Luo, Layer-by-Layer Assembled Film of Asphaltenes/ Polyacrylamide and its Stability of Water-in-Oil Emulsions: A Combined Experimental and Simulation Study, J. Phys. Chem. C, 2017, 121(8), 4332-4342.

14 A. A. Petrov and I. K. Shtof, Investigation of structure of crude oil emulsion stabilizers by means of infrared spectroscopy, Chem. Technol. Fuels Oils, 1974, 10(8), 654-657.

15 C. L. Chang and H. S. Fogler, Stabilization of asphaltenes in aliphatic solvents using alkylbenzene-derived amphiphiles. 1. Effect of the chemical structure of amphiphiles on asphaltene stabilization, Langmuir, 1994, 10(6), 1749-1757.

16 C. Jian, T. Tang and S. Bhattacharjee, Probing the effect of side-chain length on the aggregation of a model asphaltene using molecular dynamics simulations, Energy Fuels, 2013, 27(4), 2057-2067.

17 H. S. Silva, A. C. Sodero, B. Bouyssiere, H. Carrier, J. P. Korb, A. Alfarra, G. Vallverdu, D. Bégué and I. Baraille, Molecular Dynamics Study of Nanoaggregation in Asphaltene Mixtures: Effects of the N, O, and S Heteroatoms, Energy Fuels, 2016, 30(7), 5656-5664.

18 H. Rezaei, S. Amjad-Iranagh and H. Modarress, SelfAccumulation of Uncharged Polyaromatic Surfactants at Crude Oil-Water Interface: A Mesoscopic DPD Study, Energy Fuels, 2016, 30(8), 6626-6639.

19 Y. Mikami, Y. Liang, T. Matsuoka and E. S. Boek, Molecular dynamics simulations of asphaltenes at the oil-water interface: from nanoaggregation to thin-film formation, Energy Fuels, 2013, 27(4), 1838-1845.

20 F. Alvarez, E. A. Flores, L. V. Castro, J. G. Hernández, A. López and F. Vazquez, Dissipative particle dynamics (DPD) study of crude oil-water emulsions in the presence of a functionalized co-polymer, Energy Fuels, 2010, 25(2), 562567.

21 R. Skartlien, S. Simon and J. Sjøblom, A DPD study of asphaltene aggregation: The role of inhibitor and 
asphaltene structure in diffusion-limited aggregation, $J$. Dispersion Sci. Technol., 2017, 38(3), 440-450.

22 X. Song, P. Shi, M. Duan, S. Fang and Y. Ma, Investigation of demulsification efficiency in water-in-crude oil emulsions using dissipative particle dynamics, $R S C A d v$., 2015, 5(77), 62971-62981.

23 R. Skartlien, S. Simon and J. Sjöblom, DPD Molecular Simulations of Asphaltene Adsorption on Hydrophilic Substrates: Effects of Polar Groups and Solubility, $J$. Dispersion Sci. Technol., 2016, 37(6), 866-883.

24 S.-F. Zhang, L. L. Sun, J.-B. Xu, H. Wu and H. Wen, Aggregate structure in heavy crude oil: using a dissipative particle dynamics based mesoscale platform, Energy Fuels, 2010, 24, 4312-4326.

25 R. D. Groot and P. B. Warren, Dissipative particle dynamics: bridging the gap between atomistic and mesoscopic simulation, J. Chem. Phys., 1997, 107, 4423-4435.

26 Y. T. Liu, Y. R. Li and X. Wang, Spontaneous onion shape vesicle formation and fusion of comb-like block copolymers studied by dissipative particle dynamics, $R S C$ Adv. , 2017, 7(9), 5130-5135.

27 G. Dorenbos, Pore design within amphiphilic polymer membranes: linear versus Y-shaped side chain architectures, RSC Adv., 2014, 4(92), 51038-51046.

28 X. Song, P. Shi, S. Zhao, M. Duan, C. Wang and Y. Ma, Dissipative Particle Dynamics Study on the Aggregation Behavior of Asphaltenes under Shear Fields, Ind. Eng. Chem. Res., 2016, 55(33), 9077-9086.

29 C. M. Hansen, Hansen Solubility Parameters: A User's Handbook, CRC Press, Boca Raton, FL, 2007.

30 K. Shi, C. Lian, Z. Bai, S. Zhao and H. Liu, Dissipative particle dynamics study of the water/benzene/caprolactam system in the absence or presence of non-ionic surfactants, Chem. Eng. Sci., 2015, 122, 185-196.

31 S. F. Zhang, J. B. Xu, H. Wen and S. Bhattacharjee, Integration of Rotational Algorithms into Dissipative Particle Dynamics: Modeling Polyaromatic Hydrocarbons on the Meso-Scale, Mol. Phys., 2011, 109, 1873-1888.

32 F. Trejo, J. Ancheyta and M. S. Rana, Structural Characterization of Asphaltenes Obtained from Hydro Processed Crude Oils by SEM and TEM, Energy Fuels, 2009, 23, 429-439.

33 Accelrys MS Modeling 6.0, Accelrys, Inc., San Diego, CA, 2011.

34 Y. Li, Y. Guo, G. Xu, Z. Wang, M. Bao and N. Sun, Dissipative particle dynamics simulation on the properties of the oil/ water/surfactant system in the absence and presence of polymer, Mol. Simul., 2013, 39, 299-308.

$35 \mathrm{~J}$. Liu, Y. Zhao and S. Ren, Molecular dynamics simulation of self-aggregation of asphaltenes at an oil/water interface: formation and destruction of the asphaltene protective film, Energy Fuels, 2015, 29(2), 1233-1242.
36 T. F. Headen, E. S. Boek and N. T. Skipper, Evidence for asphaltene nanoaggregation in toluene and heptane from molecular dynamics simulations, Energy Fuels, 2009, 23(3), 1220-1229.

37 D. M. Sztukowski and H. W. Yarranton, Rheology of asphaltene-toluene/water interfaces, Langmuir, 2005, 21, 11651-11658.

38 J. P. Rane, V. Pauchard, A. Couzis and S. Banerjee, Interfacial rheology of asphaltenes at oil-water interfaces and interpretation of the equation of state, Langmuir, 2013, 29(15), 4750-4759.

39 Y. Li, Y. Guo, M. Bao and X. Gao, Investigation of Interfacial and Structural Properties Of CTAB at the Oil/Water Interface Using Dissipative Particle Dynamics Simulations, J. Colloid Interface Sci., 2011, 361, 573-580.

40 P. Tchoukov, F. Yang, Z. Xu, T. Dabros, J. Czarnecki and J. Sjöblom, Role of asphaltenes in stabilizing thin liquid emulsion films, Langmuir, 2014, 30(11), 3024-3033.

41 Z. Luo and J. Jiang, pH-sensitive drug loading/releasing in amphiphilic copolymer PAE-PEG: integrating molecular dynamics and dissipative particle dynamics simulations, $J$. Controlled Release, 2012, 162, 185-193.

42 J. Jestin, S. Simon, L. Zupancic and L. Barre, A Small Angle Neutron Scattering Study of the Adsorbed Asphaltene Layer in Water-in-Hydrocarbon Emulsions: Structural Description Related to Stability, Langmuir, 2007, 23, 1047110478.

43 V. Verruto and P. K. Kilpatrick, Water-in-Model Oil Emulsions Studied by Small-Angle Neutron Scattering: Interfacial Thickness and Composition, Langmuir, 2008, 24, 12807-12822.

44 Y. Mikami, Y. Liang, T. Matsuoka and E. S. Boek, Molecular Dynamics Simulations of Asphaltenes at the Oil-Water Interface: From Nanoaggregation to Thin-Film Formation, Energy Fuels, 2013, 27, 1838-1845.

45 V. J. Verruto and P. K. Kilpatrick, Water-in-model oil emulsions studied by small-angle neutron scattering: interfacial film thickness and composition, Langmuir, 2008, 24(22), 12807-12822.

46 H. Liu, Y. Li, W. E. Krause, M. A. Pasquinelli and O. J. Rojas, Mesoscopic Simulations of the Phase Behavior of Aqueous EO19PO29EO19 Solutions Confined and Sheared by Hydrophobic and Hydrophilic Surfaces, ACS Appl. Mater. Interfaces, 2012, 4(1), 87-95.

47 X. Song, S. Zhao, S. Fang, Y. Ma and M. Duan, Mesoscopic Simulations of Adsorption and Association of PEO-PPOPEO Triblock Copolymers on a Hydrophobic Surface: From Mushroom Hemisphere to Rectangle Brush, Langmuir, 2016, 32(44), 11375-11385. 